\title{
Erratum to: Patient costs of breast cancer endocrine therapy agents under Medicare Part D vs with generic formulations
}

Ann Butler Nattinger ${ }^{1,4^{*}}$, Liliana E Pezzin ${ }^{1,4}$, Emily L McGinley ${ }^{4}$, John A Charlson ${ }^{2,4}$, Tina W F Yen ${ }^{3,4}$ and Joan M Neuner ${ }^{1,4}$

\section{Erratum to: SpringerPlus (2015) 4:54}

\section{DOI 10.1186/s40064-015-0827-8}

It has come to our attention that during production of the original article, some of the column headings were missing from Tables 1 and 2 . The corrected Tables 1 and 2 can be found below. The publisher apologises for any inconvenience caused.

Table 1 Trajectory of costs under Medicare Part D for breast cancer adjuvant endocrine agents prior to generic availability

\begin{tabular}{|c|c|c|c|c|c|c|c|c|c|}
\hline \multirow[t]{4}{*}{ Medication } & \multirow{2}{*}{\multicolumn{3}{|c|}{$\begin{array}{l}\text { Monthly cost after deductible } \\
\text { Median of state mean costs }\end{array}$}} & \multirow{2}{*}{\multicolumn{3}{|c|}{$\frac{\text { Monthly cost in gap }}{\text { Median of state mean costs }}$}} & \multicolumn{3}{|c|}{ Monthly cost in catastrophic } \\
\hline & & & & & & & Median of state $\mathrm{m}$ & lean costs & \\
\hline & \multicolumn{3}{|c|}{ \$, (Range of mean costs) } & \multicolumn{3}{|c|}{ \$, (Range of mean costs) } & \multicolumn{3}{|c|}{$\$$, (Range of mean costs) } \\
\hline & 2007 & 2010 & $\begin{array}{l}\text { Change } \\
\text { (\%) }\end{array}$ & 2007 & 2010 & $\begin{array}{l}\text { Change } \\
\text { (\%) }\end{array}$ & 2007 & 2010 & $\begin{array}{l}\text { Change } \\
\text { (\%) }\end{array}$ \\
\hline Arimidex & $39.64(38.37-48.19)$ & $62.83(60.00-74.75)$ & +58 & $258.64(247.44-263.47)$ & $392.84(375.66-418.58)$ & +52 & $13.18(12.68-13.32)$ & $19.65(19.03-20.93)$ & +49 \\
\hline Aromasin & $42.57(40.72-50.94)$ & $86.50(81.51-101.08)$ & +103 & $267.56(255.21-273.62)$ & $353.98(351.86-377.07)$ & +32 & $13.66(13.07-13.79)$ & $17.70(17.59-18.85)$ & +30 \\
\hline Femara & $41.92(39.38-51.11)$ & $89.03(80.95-102.77)$ & +112 & 277.65 (249.14-282.54) & $433.42(430.46-461.42)$ & +56 & $14.16(12.96-14.34)$ & $21.67(21.55-23.07)$ & +53 \\
\hline Tamoxifen & $6.19(5.95-9.22)$ & $5.98(5.66-6.75)$ & -3 & $21.32(20.36-25.42)$ & $15.52(15.03-18.90)$ & -27 & $2.26(2.20-2.70)$ & $2.60(2.58-2.75)$ & +15 \\
\hline
\end{tabular}

\footnotetext{
*Correspondence: anatting@mcw.edu

${ }^{1}$ Division of General Internal Medicine, Medical College of Wisconsin,

8701 Watertown Plank Road, Milwaukee, WI, USA

Full list of author information is available at the end of the article
} 
Table 2 Trajectory of costs under Medicare Part D for breast cancer adjuvant endocrine agents before and after availability of generic alternatives

\begin{tabular}{|c|c|c|c|c|c|c|c|c|c|}
\hline \multirow[t]{4}{*}{ Medication } & \multirow{2}{*}{\multicolumn{3}{|c|}{$\begin{array}{l}\text { Monthly cost after deductible } \\
\text { Median of state mean costs }\end{array}$}} & \multirow{2}{*}{\multicolumn{3}{|c|}{$\frac{\text { Monthly cost in gap }}{\text { Median of state mean costs }}$}} & \multirow{2}{*}{\multicolumn{3}{|c|}{$\frac{\text { Monthly cost in catastrophic }}{\text { Median of state mean costs }}$}} \\
\hline & & & & & & & & & \\
\hline & \multicolumn{3}{|c|}{ \$, (Range of mean costs) } & \multicolumn{3}{|c|}{ \$, (Range of mean costs) } & \multicolumn{3}{|c|}{ \$, (Range of mean costs) } \\
\hline & 2007 & $2011^{a}$ & $\begin{array}{l}\text { Change } \\
(\%)\end{array}$ & 2007 & $2011^{a}$ & $\begin{array}{l}\text { Change } \\
(\%)\end{array}$ & 2007 & $2011^{a}$ & $\begin{array}{l}\text { Change } \\
(\%)\end{array}$ \\
\hline $\begin{array}{l}\text { Arimidex/ } \\
\text { Anastro- } \\
\text { zole }\end{array}$ & $39.64(38.37-48.19)$ & $9.57(8.00-11.95)$ & -76 & $258.64(247.44-263.47)$ & $38.94(31.80-78.46)$ & -85 & $13.18(12.68-13.32)$ & $3.72(3.39-6.19)$ & -72 \\
\hline $\begin{array}{l}\text { Aromasin/ } \\
\text { Exemes- } \\
\text { tane }\end{array}$ & $42.57(40.72-50.94)$ & $49.50(42.34-118.68)$ & +16 & $267.56(255.21-273.62)$ & $244.51(228.61-270.95)$ & -9 & $13.66(13.07-13.79)$ & $14.41(13.51-71.41)$ & +6 \\
\hline $\begin{array}{l}\text { Femara/ } \\
\text { Letrozole }\end{array}$ & $41.92(39.38-51.11)$ & $69.31(59.69-85.17)$ & +65 & 277.65 (249.14-282.54) & $225.13(209.10-252.23)$ & -19 & $14.16(12.96-14.34)$ & $38.53(33.70-57.15)$ & +172 \\
\hline Tamoxifen & $6.19(5.95-9.22)$ & $5.54(5.12-6.89)$ & -11 & $21.32(20.36-25.42)$ & $13.17(12.44-21.11)$ & -38 & $2.26(2.20-2.70)$ & $2.73(2.50-3.47)$ & +21 \\
\hline
\end{tabular}

${ }^{\text {a }}$ First year generic formulation was available to Medicare Part D beneficiaries; generic cost provided

\section{Author details}

${ }^{1}$ Division of General Internal Medicine, Medical College of Wisconsin, 8701

Watertown Plank Road, Milwaukee, WI, USA. ${ }^{2}$ Division of Hematology-Oncology, Medical College of Wisconsin, 8701 Watertown Plank Road, Milwaukee, WI, USA. ${ }^{3}$ Division of Surgical Oncology, Medical College of Wisconsin, 8701 Watertown Plank Road, Milwaukee, WI, USA. ${ }^{4}$ Center for Patient Care and Outcomes Research, Medical College of Wisconsin, 8701 Watertown Plank Road, Milwaukee, WI 53226, USA.

Published online: 21 September 2015 